\section{Estudo Morfológico do desenvolvimento da cartilagem quadrangular do nariz e implicações nas cirurgias septoplásticas}

Paulo S. G. Pereira, MD, João K. Kajiwara, MD, PHD, Marcos Grellet, MD, PHD

Resumo

$\mathbf{I}$ ntrodução: Este trabalho é um estudo morfológico do desenvolvimento da cartilagem quadrangular humana ao longo da vida. Para tal retiramos cartilagens quadrangulares em bloco com as demais cartilagens do nariz, utilizando a técnica de rinoplastia aberta, em cadáveres autopsiados no Serviço de Patologia do Hospital das Clínicas da Faculdade de Medicina de Ribeirão Preto - USP e no serviço de verificação de óbitos do interior, em Ribeirão Preto. Forma de estudo: Experimental. Métodos: As peças anatômicas foram cortadas em dois pontos dividindo-as em três partes, com relação ao eixo caudo-cefálico correspondendo à borda livre da cartilagem, terço caudal (posição 1), médio (posição 2) e cefálico (posição 3). Tem relação com a placa perpendicular do etmóide e volmer. As amostras foram fixadas em formol a 10\% e incluídas em parafina. Foram examinados cortes histológicos de $7 \mu \mathrm{m}$ de espessura e corados por hematoxilina e eosina (HE) e Alcian Blue. Em cada posição, três diferentes locais, em relação ao eixo ântero-posterior do nariz: Terço superior (local 1), terço médio (local 2) e terço inferior (local 3) foram estudados sobre Microscopia Óptica, com métodos fotométricos, histológicos e morfométricos. Resultado: Mostra que o tecido cartilaginoso apresenta maior velocidade de crescimento até cinco anos de idade. A menor velocidade de crescimento ocorre a partir dos oito anos de idade. Não ocorreu diferença de atividade metabólica entre as diversas regiões estudadas. Conclusões: As intervenções cirúrgicas no septo cartilaginoso podem ser realizadas após os cinco anos de idade. A idade inicial mais adequada para as cirurgias do septo nasal ocorreria aos oito anos.

\section{Morphologic study of the development of the human nose quadrilateral cartilage and implications in the septoplastic surgery}

Palavras-chave: septo nasal, cartilagem quadrilateral, crescimento do septo.

Key words: septum of the nose, quadrilateral cartilage, growth of the septum.

\section{Summary}

\begin{abstract}
Introduction: The present paper is a morphological study of human quadrilateral cartilage including 0 to 66 years old subjects. The quadrilateral cartilage was removed in block with the others nasal cartilages. The open rhinoplasty was the approach used to remove the cartilage from deceased patients autopsied at Serviço de Patologia do Hospital das Clínicas da Faculdade de Medicina de Ribeirão Preto - USP São Paulo and Serviço de Verificação de óbitos do Interior, at Ribeirão Preto, São Paulo. Study design: Experimental. Methods: The anatomical parts were cut into three peaces, each corresponding to the: caudal position (position 1), mid (position 2) and cefalic (position 3 ) thirds. After fixing and embedding the samples were cut in $7 \mu \mathrm{m}$ slice, staining them by Hematoxylin \& Eosin or Alcian Blue. Each position was divided in three locals: supeior anterior), (local 1), mid (local 2) and Inferior (posterior), (local 3) thirds and studied in optical microscopy by photometric, histological and morphometric methods. Results: The main results were that quadrilateral cartilagenous tissue have the highest growth speed until the age 5 years. After age of 8 years, there is a decrease in this speed. Conclusion: Nasoseptal surgeries can be done after age of 5 years and the most adequate age to do these surgeries is 8 years old. There was no diference among diferents parts of the septal cartilage studied.
\end{abstract}




\section{INTRODUÇÃO}

A obstrução nasal na infância é queixa importante na clínica otorrinolaringológica. Neste período é necessário que se dê um enfoque especial para ela, pois é o período de franco crescimento e desenvolvimento da face e do nariz. Entre as causas mais significativas da obstrução nasal na infância estão os desvios do septo que ocorrem em cerca de $20 \%$ da população. Em 25\% dos portadores esta irregularidade é suficiente para produzir uma resistência importante ao fluxo de $\operatorname{ar}^{1}$. A cirurgia septal na infância surgiu com o interesse em restabelecer a função nasal normal, ajudar o desenvolvimento normal do nariz, prevenir subdesenvolvimento da columela e prevenir deformidades da ponta nasal. ${ }^{2}$ No início, a principal cirurgia para corrigir os desvios do septo nasal foi a Ressecção Submucosa do Septo (RSM) idealizadas por Killian \& Free ${ }^{1}$. Acreditava-se que esta cirurgia destruía os chamados centros de crescimento da cartilagem septal com conseqüente impacto no crescimento do septo nasal e do crescimento facial, fazendo com que estas cirurgias fossem adiadas até os 15 ou 16 anos de idade. Surgiram cirurgiões contra $^{2,3,4,5,6,7,8,9,10,11,12}$ e a favor do procedimento na infância ${ }^{13,14,15,16,17,18,19,20}$. Assim, vários estudos concentraramse na identificação dos centros de crescimento da cartilagem septal com o intuito de amenizar os problemas decorrentes da cirurgia e, dessa forma, tornar possível a correção das deformidades antes da puberdade. Nosso trabalho visa determinar o comportamento de diferentes regiões da cartilagem quadrangular do nariz humano durante o crescimento e sugerir implicações nas condutas cirúrgicas septoplásticas na infância.

\section{MATERIAL E MÉTODO}

Foram retiradas 44 cartilagens septais de cadáveres humanos submetidos a exame necroscópico. Restringiu-se a amostra ao material advindo de indivíduos brancos, do sexo masculino e faixa etária entre cinco dias a 66 anos, totalizando 12 cartilagens. A cartilagem septal foi retirada em conjunto com todo arcabouço cartilaginoso através da via de acesso por rinoplastia aberta preconizada por Goodman ${ }^{21,22,23,24}$ e por Anderson ${ }^{25}$; foi idealizada uma técnica de reconstrução da pirâmide nasal, usando como matériaprima papelão e algodão. A sutura da incisão foi realizada com cat gut cromado 0000, não deixando nenhuma deformidade nasal no pós-ressecção. As peças retiradas foram imediatamente fixadas em solução de formalina a $10 \%$. Reconhecida as relações anatômicas, as peças foram aparadas com lâmina de bisturi mediante o estabelecimento de dois planos de secção frontais: um na transição do terço caudal e médio e outro na transição do terço médio e cefálico. O resultado foi a definição do bloco em três peças: terço caudal (posição 1), terço médio posição 2) e terço cefálico (posição 3). Por sua vez, cada posição foi subdividida em locais no eixo ântero-posterior: terço anterior superior (local 1), terço médio, centro, (local 2), terço posterior, inferior (local 3), assim as três diferentes posições divididas em três diferentes locais constituíram-se nas 9 regiões da cartilagem estudada (Figura 1).

Preparação histológica: decorridas 24 horas de imersão na solução fixadora, as peças foram submetidas à desidratação em baterias de soluções de concentração crescente de etanol (50\%, 60\%, 70\%, 80\%, 90\%, 95\%, 100\% e 100\%)* e diafanizadas em solução de benzeno mais etanol ( $1: 1 \mathrm{v} / \mathrm{v})$, benzeno $100 \%$ e incluídas em parafina. Das peças obtivemos blocos que foram cortados em fatias de $7 \mu \mathrm{m}$ de espessura, que foram colocadas em lâminas de vidro, desparafinados com solução de xilol e rehiadratados

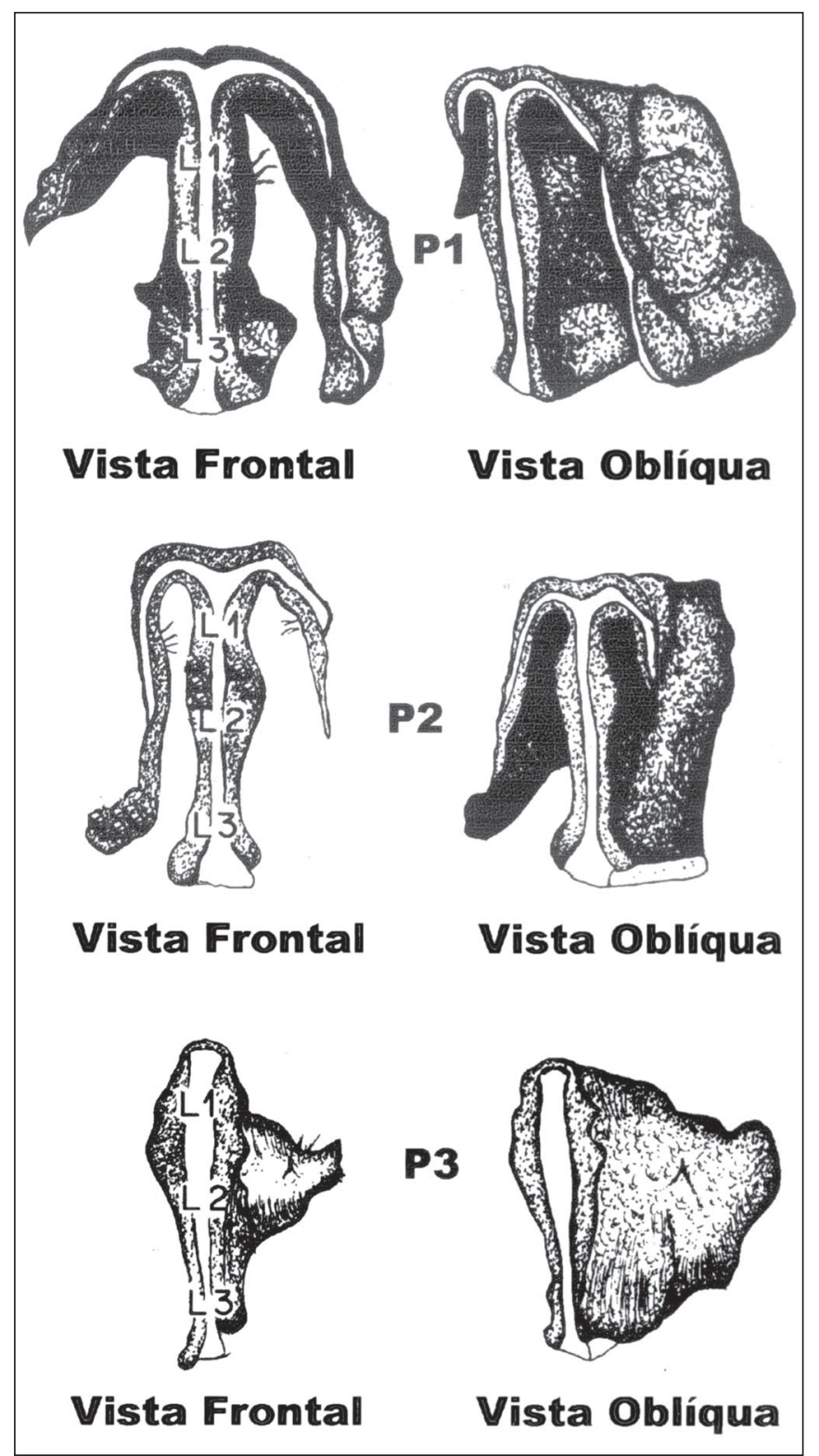

Figura 1. Regiões que definem as posições e locais deste estudo. 
imergindo as lâminas em xilol mais etanol (1:1 v/v), em bateria de concentrações decrescentes de etanol (100\%, 100\%, 95\%, 90\%, 80\%, 70\%, 60\%)*. Após a rehidratação, metade das lâminas de cada posição e local de cada caso foi corada pela técnica de hematoxilina e eosina (HE) e outra metade pelo azul de Alcian. As lâminas contendo os cortes foram rehidratadas seguindo o caminho reverso da hidratação, diafanizadas com benzeno e montadas em permount.

\section{Três estudos foram aplicados:}

1. Análise histológica: foi realizada nas lâminas coradas por HE, com microscópio Zeiss, com objetiva de 40X. Deu-se atenção basicamente às observações das características de crescimento encontradas em tecido cartilaginoso.

2. Densitometria óptica: foi realizada nas lâminas coradas pelo azul de Alcian com o objetivo de determinar variações na matriz cartilaginosa. Este corante interage com as glicosaminoglicanas poliônicas sulfatadas presentes na matriz ${ }^{26}$ e desta forma a concentração das mesmas refletiria a atividade metabólica da cartilagem e esta atividade poderia ter relação com os fenômenos de crescimento da mesma. A densitometria óptica do azul de alcian pode dar noções das concentrações relativas entre as várias regiões e entre várias idades dentro de uma mesma região pela aplicação da lei de BeerLambert. Esta lei baseia-se no fato de que em meios onde uma substância esteja homogeneamente diluída, a absorbância de luz monocromática dependerá diretamente do comprimento do trajeto de luz dentro da solução, diretamente da concentração do soluto e inversamente da área de projeção da luz. Fixados o comprimento do trajeto e a área de projeção, a absorbância de luz monocromática será proporcional à concentração da substância estudada, segundo a fórmula:

$$
\alpha=\log \frac{\mathrm{I}}{\mathrm{I}_{0}}
$$

Onde: $\alpha=$ absorbância

$\log =\log$ aritmo na base 10

$\mathrm{I}=$ intensidade de luz detectada interpondo-se o objeto

$\mathrm{I}_{0}=$ intensidade de luz detectada sem interposição do objeto.

A absorbância não é linearmente relacionada à quantidade de luz absorvida pela substância de interesse e, portanto, meios heterogêneos como os encontrados em preparações histológicas são sujeitos a erros distribucionais. Dessa forma, ao tomarmos a intensidade de luz em uma área heterogeneamente corada de uma preparação o valor de I será a média dos valores de intensidade luminosa nos inúmeros pontos que envolvem somas anteriores. A maneira mais prática de efetuar uma correção deste tipo de erro é dividir a região de interesse em inúmeras regiões pequenas onde a heterogeneidade seja pequena, tomar o valor de absorbância em cada uma delas e realizar a média de todas as absorbâncias obtidas. O programa Image tool do University of Texas Health Science Center at San Antônio foi o programa adotado nas medidas de densidade óptica. O padrão de calibração de distâncias lineares foi uma lâmina graduada em traços de $10 \mu \mathrm{m}$ da Zeiss. As densidades ópticas foram tomadas na matriz cartilaginosa em cada posição e local da região subpericondral de um lado a região subpericondral do outro lado, utilizando como monocromatizador o filtro presente no conjunto número 9 da Zeiss. As medidas de $\mathrm{I}_{0}$ foram feitas medindo-se a intensidade luminosa presente em regiões da lâmina que não contivessem nenhum corte. Nas comparações de densidades ópticas, foram feitas análises de regressão linear em cada posição e em cada local, tomando-se a idade dos indivíduos estudados como variável independente.

3. Estudo Morfométrico: As medidas morfométricas foram realizadas nas lâminas coradas por HE. O sistema de captura e de análise de imagens foi o mesmo das medidas de densidade óptica. Foram medidas as espessuras da cartilagem e do pericôndrio em cada uma das posições e respectivos locais e o comprimento do septo nas três posições. Foram realizadas 10 medidas em cada uma das regiões e tomada a média destas 10 medidas. Na análise da variação da espessura da cartilagem foi aplicada a regressão logarítmica nos valores obtidos, pois esta função foi a que melhor se ajustou na maioria dos casos.

A função logarítmica é representada por:

$$
\varepsilon=a \ln \imath+b
$$

Onde: $\boldsymbol{\varepsilon}$ é a espessura observada da cartilagem. a e b são constantes. $\mathfrak{\imath}$ é a idade em anos.

Obtidas as equações que representam a espessura da cartilagem em relação à idade em anos, foi calculada a velocidade de aumento da espessura simplesmente utilizando a função derivada da fórmula logarítmica obtendo-se $\mathbf{v}=\mathbf{a} \mathbf{~}^{-\mathbf{1}}$, onde $\mathbf{v}=$ velocidade de aumento de espessura da cartilagem, a é a mesma constante e $\mathbf{\imath}$ é a idade em anos. Na análise da variação da espessura do pericôndrio foi aplicada a regressão polinomial de grau 3, pois esta função foi a que melhor se ajustou na maioria dos casos. A função polinomial de grau 3 é representada por: $\boldsymbol{\varepsilon}=\mathbf{a} \times \mathbf{l}^{3}+\mathbf{b} \times \mathbf{l}^{2}+\mathbf{c} \mathbf{x} \mathbf{l}+\mathbf{d}$, onde: 
$\boldsymbol{\varepsilon}$ é a espessura do pericôndrio, $\mathbf{a}, \mathbf{b}, \mathbf{c}$ e $\mathbf{d}$ são constantes, $\mathbf{l}$ é a idade em anos. Portanto, as curvas obtidas representam sempre um ponto de máximo. As idades onde a espessura máxima do pericôndrio é atingida são aquelas onde a primeira derivada da função obtida acima fica com valor igual a zero e segunda derivada com valor menor que zero. Como se trata de uma função polinomial de grau 3, ela sempre admite a primeira e a segunda derivação onde se obtém duas equações: A primeira derivada é definida por $\boldsymbol{\varepsilon}^{\prime}=\mathbf{3} \times \mathbf{a} \times \boldsymbol{t}^{2}+\mathbf{2} \mathbf{x} \mathbf{b} \mathbf{x} \mathbf{t}+\mathbf{c}$ e a segunda é definida por: $\boldsymbol{\varepsilon} "=\mathbf{6} \mathbf{x} \mathbf{a} \mathbf{x} \mathbf{\imath} \mathbf{2} \mathbf{x} \mathbf{b}$, onde $\mathbf{a}, \mathbf{b}, \mathbf{c}$ e $\mathbf{l}$ são as mesmas variáveis da equação do polinômio de regressão.

Nas comparações entre valores de espessura e do comprimento da cartilagem quadrangular, os pontos foram ajustados para uma curva de regressão logarítmica e foram considerados como pontos de redução na velocidade de crescimento à idade onde a derivada da curva obtida fosse igual a 1. O programa utilizado em todas as análises estatísticas e na geração de gráficos foi o Excel versão 7 da Microsoft.

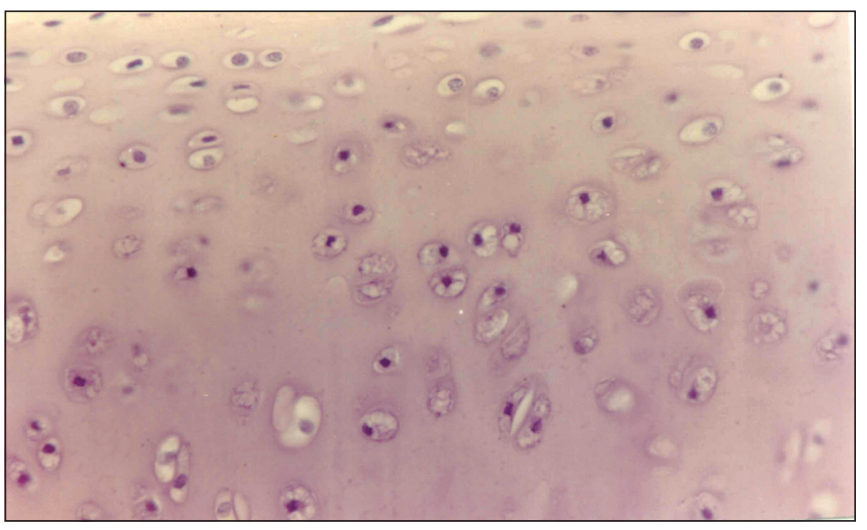

Figura 2. Indivíduo de 09 meses de idade. Zona de Transição, figuras de Mitose e Hipertrofia Celular.

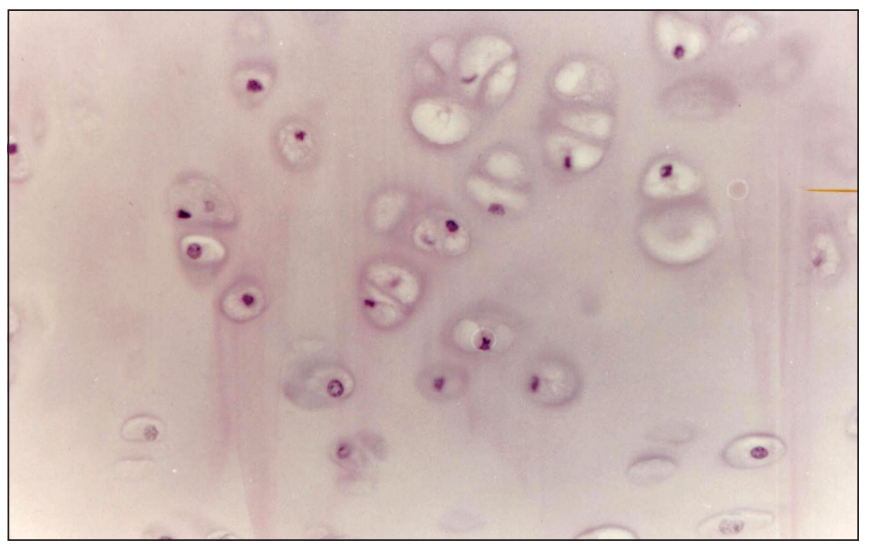

Figura 3. Indivíduo de 06 anos de idade, Hipertrofia Celular, Formação de Grupos Isógenos

\section{RESULTADOS}

1. Observações da histologia da cartilagem septal: Em cartilagens muito jovens como em indivíduos de nove meses observamos um grande número de células jovens na periferia, zona de transição bem nítida; há também grande número de células maduras no centro e formação de poucos grupos isógenos (Figura 2 região P1L1). Em indivíduos com seis anos de idade, quando esperamos uma menor velocidade de crescimento, observamos características de cartilagem madura como: poucas células na periferia, zona de transição pouco nítida, menor número de células no centro da cartilagem e formação de grupos isógenos em maior número (Figura 3 região P1L2). O mesmo ocorre em indivíduos de 12, 15 e 37 anos.

2. Densitometria óptica: A densidade óptica diminuiu significativamente em todas as regiões, com exceção do local 2, da posição 2 que corresponde ao centro geométrico da cartilagem (Tabela 1). Não ocorreram diferenças significativas entre os coeficientes angulares nas regiões onde ocorreu diminuição da densidade óptica, nem nos valores de intercepto (Figuras 4, 5 e 6).

3. Morfometria: Os parâmetros constantes da curva logarítmica, aplicados sobre a medida da espessura da cartilagem, assim como seus níveis de significância estão apresentados na Tabela 2 (Figuras 7, 8 e 9).

Nota-se que o modelo logarítmico não se ajustou para as regiões: P2L2, P3L1, P3L2 e P3L3, não sendo possível com a utilização do mesmo inferir sobre o comportamento da espessura da cartilagem nas diversas idades. Com estes parâmetros em mãos foi possível inferir os valores médios esperados de espessura de cartilagem para idades entre 1 e 15 anos, e os valores de velocidade de crescimento esperados para as mesmas idades (Tabela 3).

Tabela 1. Parâmetros obtidos da regressão linear aplicada aos valores de densitometria nas várias idades estudadas.

\begin{tabular}{lcccc}
\hline & $\mathrm{a}$ & $\mathrm{b}$ & $\mathrm{p}$ & \\
\hline P1L1 & $-0,02 \pm 0,01$ & $4,60 \pm 0,31$ & $4,8 \%$ & $*$ \\
P1L2 & $-0,03 \pm 0,01$ & $4,44 \pm 0,21$ & $0,2 \%$ & ** \\
P1L3 & $-0,02 \pm 0,01$ & $4,38 \pm 0,18$ & $1,0 \%$ & $\star \star$ \\
P2L1 & $-0,02 \pm 0,01$ & $3,77 \pm 0,24$ & $1,9 \%$ & * \\
P2L2 & $-0,01 \pm 0,01$ & $3,91 \pm 0,24$ & $12,5 \%$ & $\mathrm{NS}$ \\
P2L3 & $-0,02 \pm 0,01$ & $4,51 \pm 0,20$ & $1,9 \%$ & $*$ \\
P3L1 & $-0,02 \pm 0,01$ & $4,00 \pm 0,27$ & $2,7 \%$ & $*$ \\
P3L2 & $-0,03 \pm 0,01$ & $4,31 \pm 0,20$ & $0,3 \%$ & ** \\
P3L3 & $-0,02 \pm 0,01$ & $4,60 \pm 0,19$ & $1,5 \%$ & *
\end{tabular}

$a=$ coeficiente angular da reta de regressão

$\mathrm{b}=$ valor previsto na idade de 0 anos

$\mathrm{p}=$ significância

* = significante ao nível de 5\%

** = significante ao nível de $1 \%$

NS $=$ Não Significante 


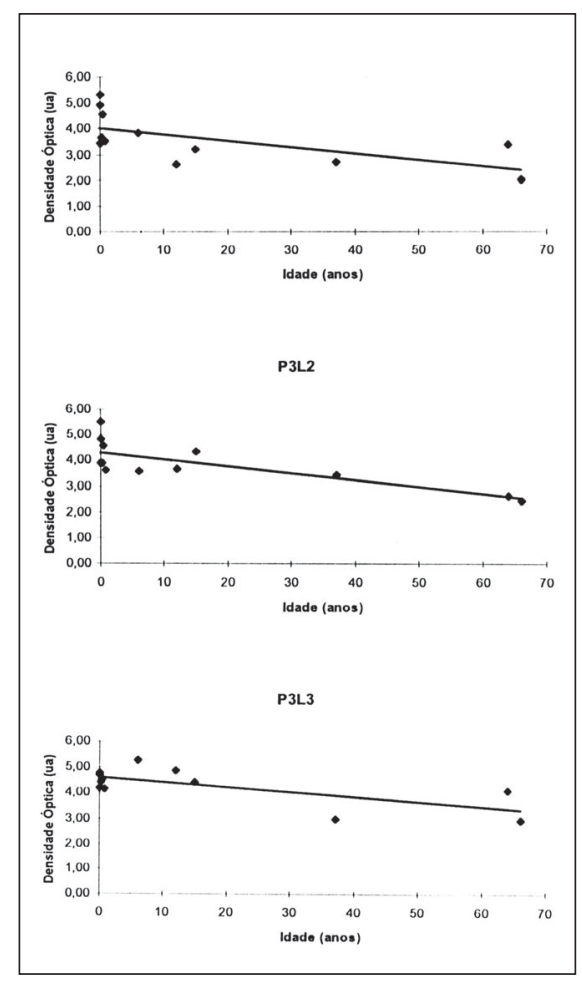

Figura 4. Valores observados e retas de regressão na posição 1

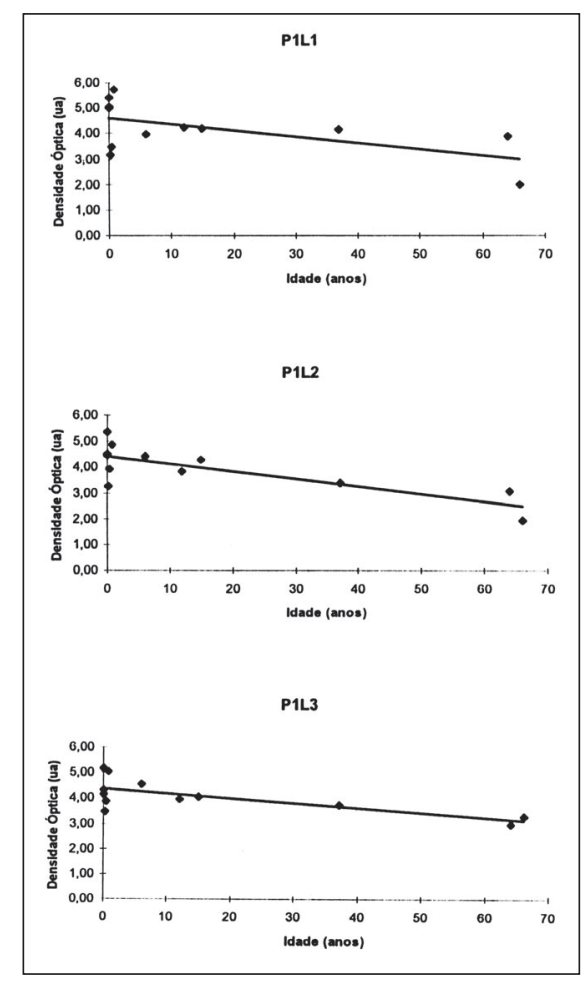

Figura 5. Valores observados e retas de regressão na posição 2

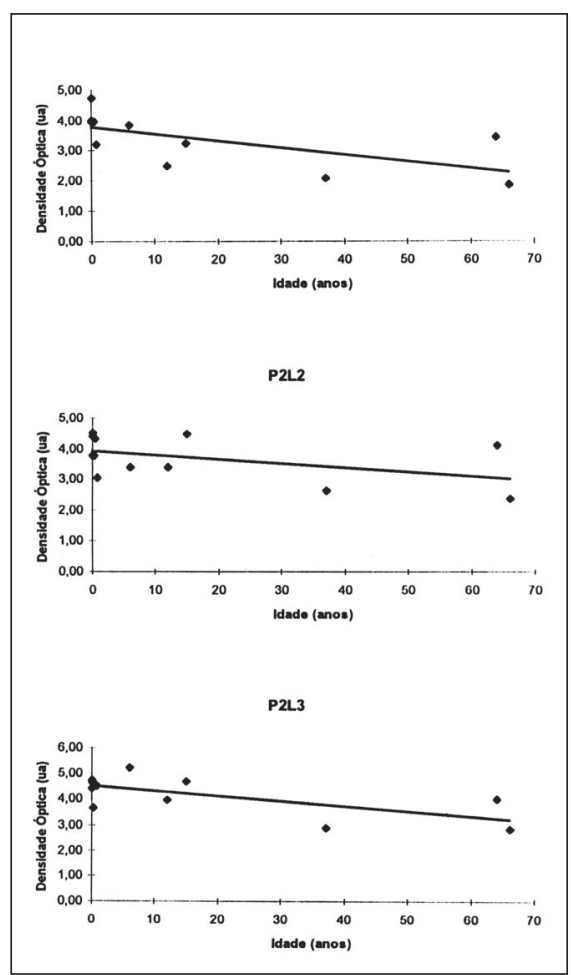

Figura 6. Valores observados e retas de regressão na posição 3 .

Tabela 2. Parâmetros da curva logarítmica obtida a partir dos dados da Tabela 1.

\begin{tabular}{ccccccccccc}
\hline & P1L1 & P1L2 & P1L3 & P2L1 & P2L2 & P2L3 & P3L1 & P3L2 & P3L3 \\
\hline $\mathrm{a}$ & 0,06 & 0,08 & 0,12 & 0,08 & 0,02 & 0,06 & 0,08 & 0,01 & $-0,01$ \\
& 0,01 & 0,01 & 0,04 & 0,02 & 0,03 & 0,01 & 0,04 & 0,02 & 0,03 \\
\hline $\mathrm{b}$ & 0,063 & 0,76 & 1,00 & 0,82 & 1,01 & 0,86 & 1,13 & 1,19 & 1,06 \\
& 0,03 & 0,03 & 0,13 & 0,07 & 0,09 & 0,04 & 0,11 & 0,07 & 0,10 \\
\hline $\mathrm{p}$ & $0,01 \%$ & $<0,01 \%$ & $2,13 \%$ & $0,58 \%$ & $40,2 \%$ & $0,15 \%$ & $5,23 \%$ & $77,4 \%$ & $87,0 \%$ \\
\hline & $* *$ & $* *$ & $*$ & $* *$ & NS & $* *$ & NS & NS & NS \\
\hline
\end{tabular}

$\mathrm{a}=$ dado em $\mu \mathrm{m} \times \mathrm{ano}^{-1}$

$\mathrm{b}=\mu \mathrm{m}$

$p=\%$

As velocidades em vermelho são aquelas em que o crescimento em um ano é maior que $2 \%$ da espessura, as em azul são aquelas em que o crescimento é maior que 1\% da espessura e as em verde são aquelas em que o crescimento é menor que 1\%. As velocidades em cinza são dos locais e posições onde o ajuste pelo logarítmico não é significativo e, portanto, não é possível inferir a velocidade de aumento de espessura.

Sobre os valores obtidos das medidas do comprimento médio da cartilagem quadrangular em cada posição, foi aplicada a análise de regressão logarítmica que mostrou que este modelo de regressão ajusta bem para o estudo
** = Significante ao nível de $1 \%$

* = Significante ao nível de $5 \%$

NS = Não Significante do comprimento do septo cartilaginoso com a idade (Tabela 4) (Figura 10).

Com estes parâmetros em mãos foi possível inferir os valores médios esperados de comprimento para as idades entre 1 e 15 anos e os valores de velocidade de aumento de comprimento esperados para as mesmas idades (Tabela 5).

Sobre os valores obtidos das medidas da espessu-

ra do pericôndrio em cada posição foi aplicada a análise de regressão polinomial de grau 3, que foi a que se ajustou melhor no estudo da variação da espessura do pericôndrio em relação a idade (Tabela 6). 


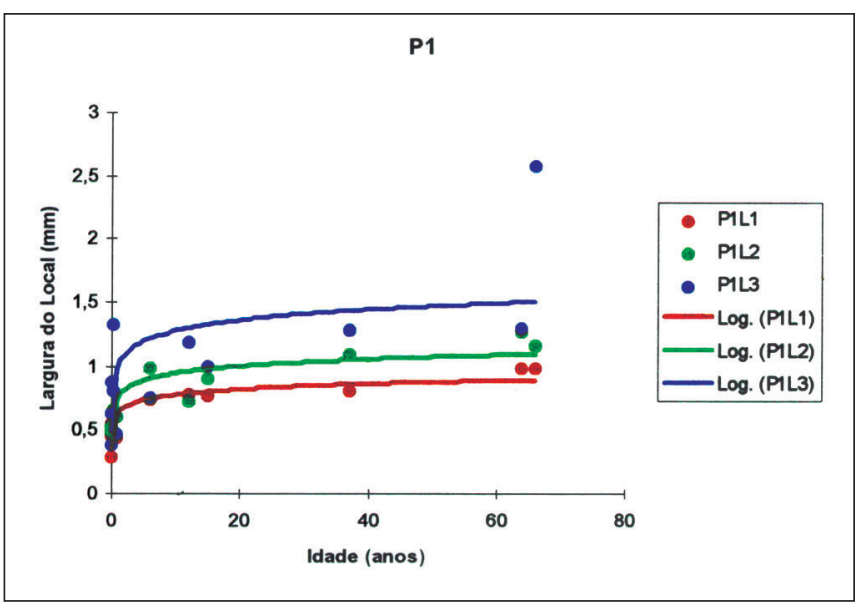

Figura 7. Valores observados da espessura média da cartilagem quadrangular na posição 1 .

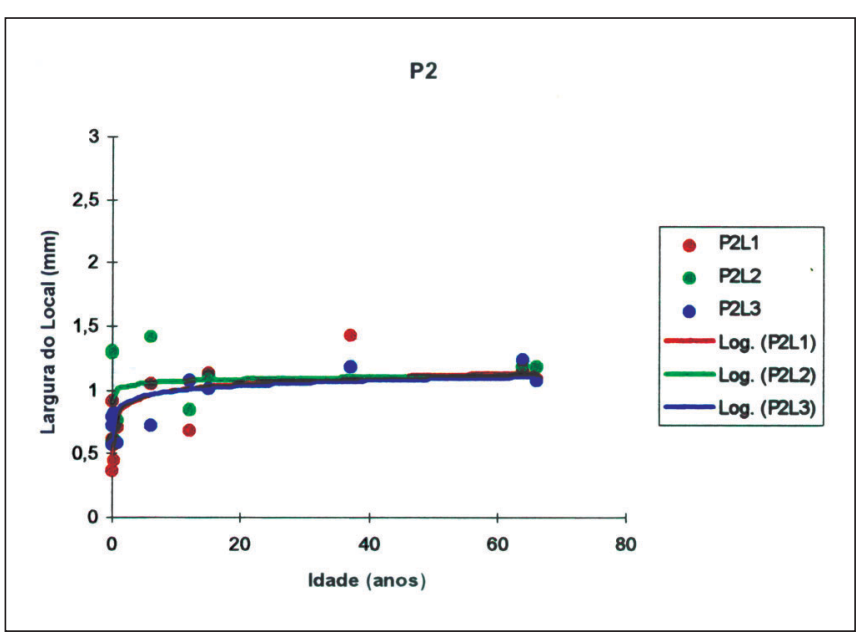

Figura 8. Valores observados de espessura média da cartilagem quadrangular na posição 2 .

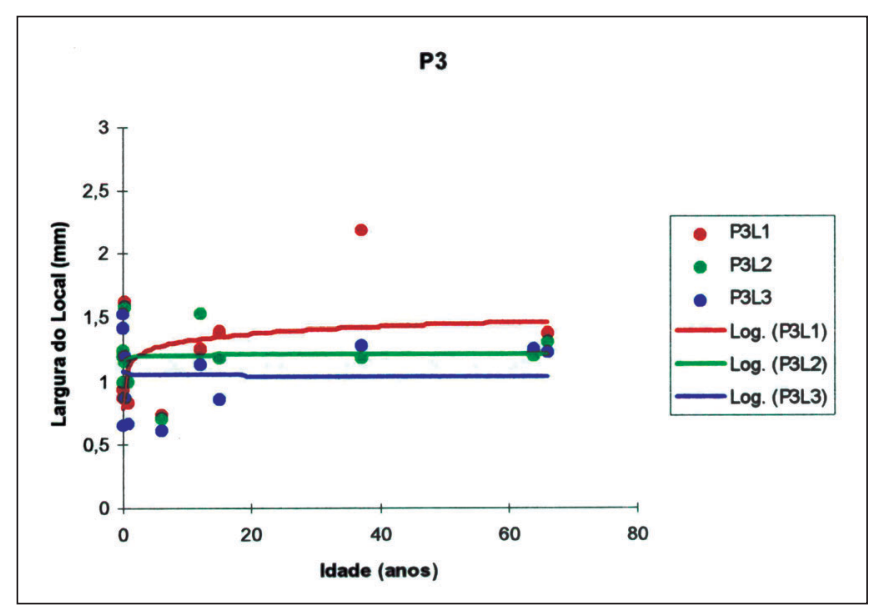

Figura 9. Valores observados de espessura média da cartilagem quadrangular na posição 3 .

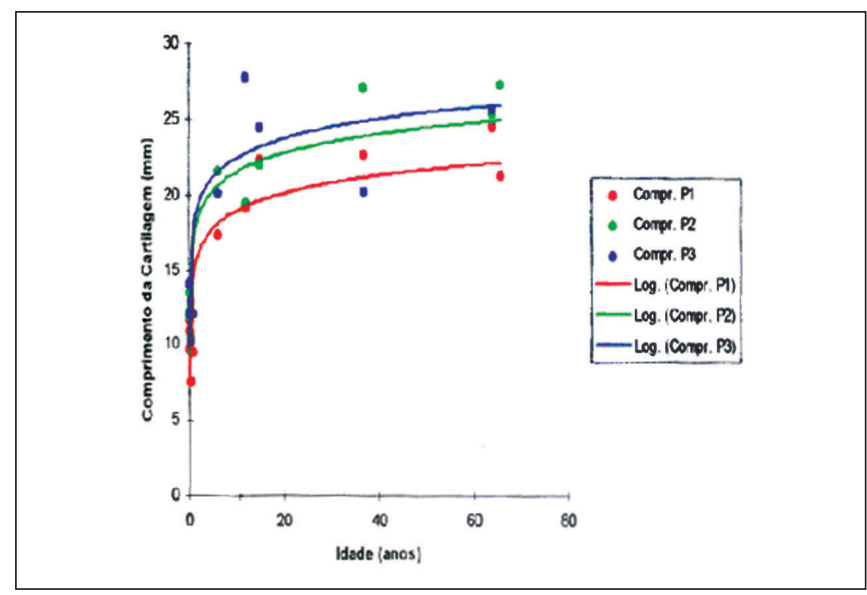

Figura 10. Valores observados de comprimento médio da cartilagem quadrangular nas posições 1,2 e 3 .

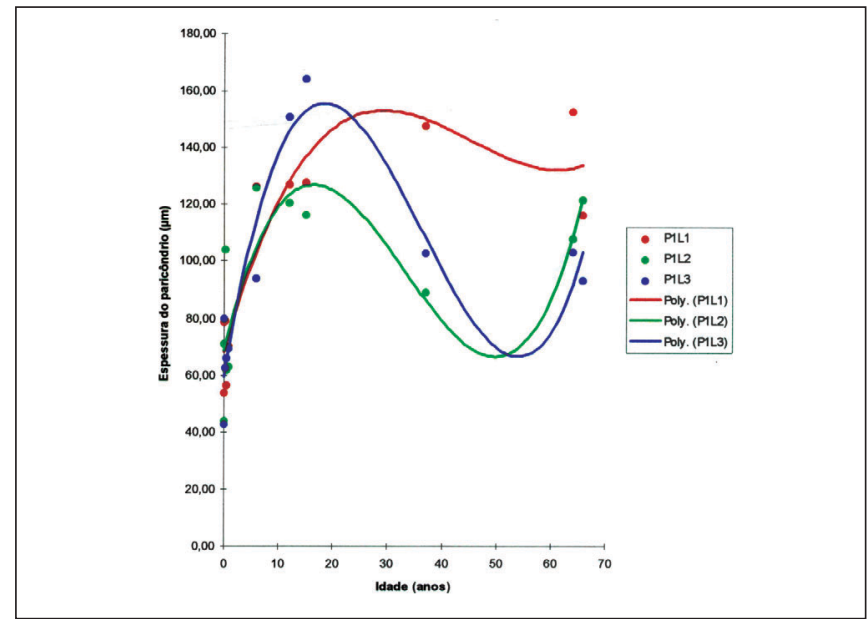

Figura 11. Valores obtidos de espessura média do pericôndrio em P1L1, p1L2 e P1L3. Os círculos representam os valores observados e as linhas são as curvas inferidas por regressão polinomial de grau 3.

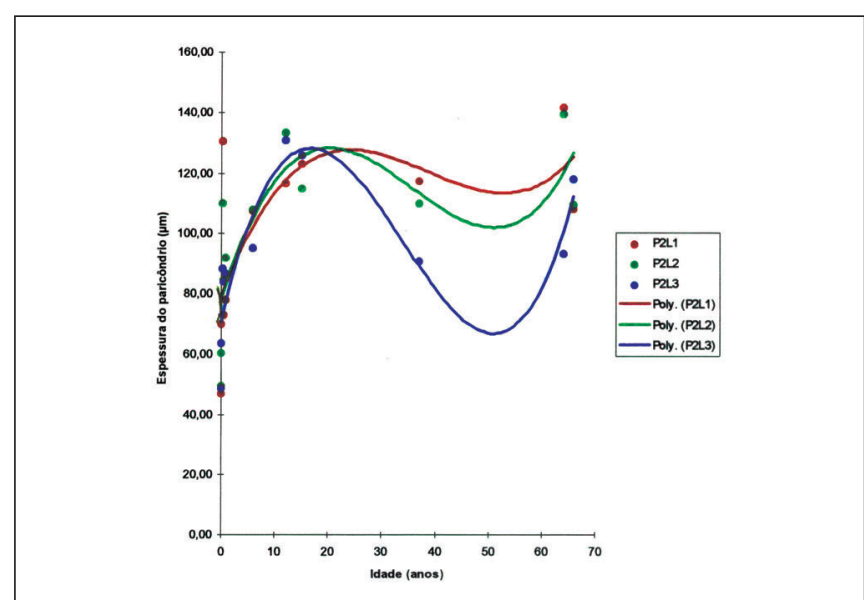

Figura 12. Valores obtidos de espessura média do pericôndrio em P2L1, P2L2 e P2L3. Os círculos representam os valores observados e as linhas são as curvas inferidas por regressão polinominal de grau 3. 


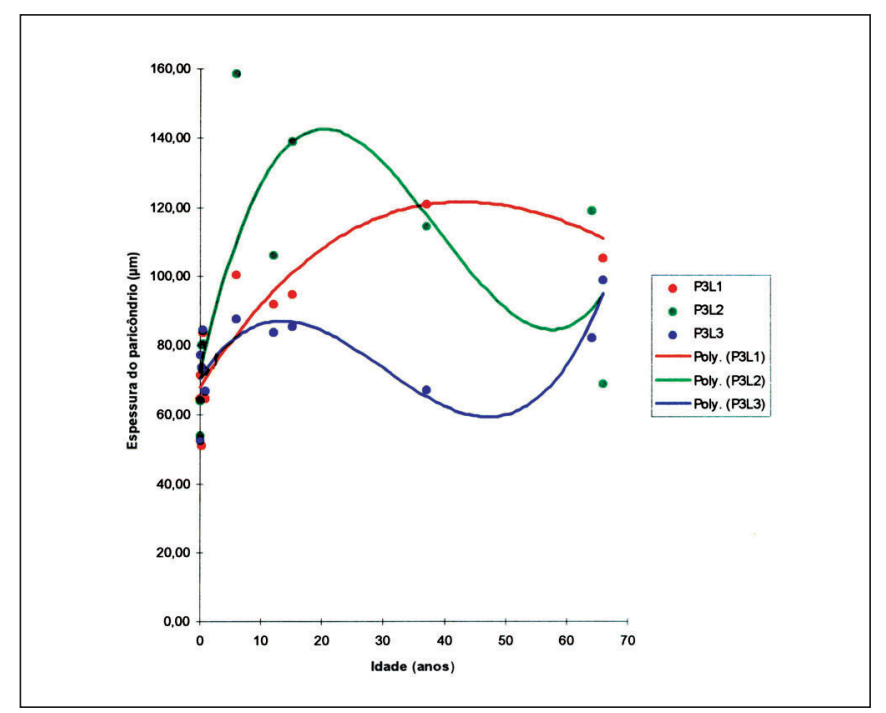

Figura 13. Valores obtidos de espessura média do pericôndrio em P3L1, P3L2, e P3L3. Os círculos representam os valores observados e as linhas são as curvas inferidas por regressão polinominal de grau 3 .
Foi significante em nível de 1\% em P1L1, P1L3, P2L3 e P3L1 e ao nível de 5\% em P1L2. Obtidos estes parâmetros foi possível determinar as idades, em cada posição e local, o pericôndrio atinge sua espessura máxima (Figuras 11, 12 e 13).

\section{DISCUSSÃO}

A identificação de áreas de maior potencial de crescimento da cartilagem quadrangular ou dos "centros de crescimento" constitui a principal preocupação para os rinocirurgiões, pois conhecendo essas áreas as cirurgias podem ser realizadas com maior segurança. Paralelamente, o conhecimento do padrão de crescimento septal é de fundamental importância, pois sabendo-se como este tecido se desenvolve, pode-se determinar a idade mais adequada para a intervenção. Em resumo, é essencial, responder a questões como: a partir de que idade é mais seguro operar? Que área da cartilagem quadrangular é mais vulnerável à intervenção cirúrgica? A cirurgia septal na

Tabela 3. Valores de velocidade de aumento de espessura esperados a partir dos parâmetros das curvas da Tabela 2, nas idades de 1 a 15 anos, nas diversas posições e locais.

\begin{tabular}{|c|c|c|c|c|c|c|c|c|c|c|c|c|c|c|c|}
\hline & 1 & 2 & 3 & 4 & 5 & 6 & 7 & 8 & 9 & 10 & 11 & 12 & 13 & 14 & 15 \\
\hline P1L1 & 62,6 & 31,3 & 20,9 & 15,7 & 12,5 & 10,4 & 8,9 & 7,8 & 7 & 6,3 & 5,7 & 5,2 & 4,8 & 4,5 & 4,2 \\
\hline P1L3 & 119,1 & 59,6 & 39,7 & 29,8 & 23,8 & 19,9 & 17 & 14,9 & 13,2 & 11,9 & 10,8 & 9,9 & 9,2 & 8,5 & 7,9 \\
\hline P2L2 & 24,4 & 12,2 & 8,1 & 6,1 & 4,9 & 4,1 & 3,5 & 3,1 & 2,7 & 2,4 & 2,2 & 2 & 1,9 & 1,7 & 1,6 \\
\hline P2L3 & 58,6 & 29,3 & 19,5 & 14,6 & 11,7 & 9,8 & 8,4 & 7,3 & 6,5 & 5,9 & 5,3 & 4,9 & 4,5 & 4,2 & 3,9 \\
\hline P3L1 & 79 & 39,5 & 26,3 & 19,8 & 15,8 & 13,2 & 11,3 & 9,9 & 8,8 & 7,9 & 7,2 & 6,6 & 6,1 & 5,6 & 5,3 \\
\hline P3L2 & 6,8 & 3,4 & 2,3 & 1,7 & 1,4 & 1,1 & 1 & 0,8 & 0,8 & 0,7 & 0,6 & 0,6 & 0,5 & 0,5 & 0,5 \\
\hline
\end{tabular}

Tabela 4. Parâmetros da curva logarítmica obtida a partir dos dados da Tabela 6.

\begin{tabular}{cccc}
\hline & $\mathrm{P} 1$ & $\mathrm{P} 2$ & $\mathrm{P} 3$ \\
\hline $\mathrm{a}$ & $1,01 \pm 0,003$ & $1,01 \pm 0,003$ & $1,01 \pm 0,003$ \\
$\mathrm{~b}$ & $11,66 \pm 0,100$ & $13,83 \pm 0,078$ & $14,44 \pm 0,093$ \\
$\mathrm{p}$ & $0,42 \%$ & $0,13 \%$ & $0,45 \%$ \\
\hline & $\star \star$ & $* \star$ & $* \star$ \\
\hline
\end{tabular}

$\mathrm{a}=$ dado em $\mathrm{mm} \times$ ano-

$\mathrm{b}=\mathrm{mm}$

$\mathrm{p}=\%$ infância, mesmo com técnica conservadora afeta o crescimento nasal? Realmente deve-se esperar até a idade de 15 ou 16 anos para realizar uma septoplastia? Mosher (apud Vetter) ${ }^{27}$ postulou dois centros de crescimento na cartilagem septal humana depois da idade de oito anos: a primeira estaria localizada acima da área da pré-maxila e a segunda na metade superior da borda septal posterior. Klaff ${ }^{28}$ relatou que na região da asa da pré-maxila a cartilagem quadrangular é fina e também o centro chefe do crescimento da mesma. White ${ }^{13}$ apontou, como centro de crescimento, a junção da cartilagem lateral inferior com

Tabela 5. Valores esperados de velocidades de aumento da curves da Tabela 6, as idades de 1 a 15 anos, nas diversas posições e locais. As velocidades em vermelho são aquelas em que o aumento em um ano é maior que $2 \%$ do comprimento, as em azul são aquelas em que o aumento é maior que $1 \%$ do comprimento e as em verde são aquelas em que o aumento é menor que $1 \%$.

\begin{tabular}{lccccccccccccccc}
\hline & 1 & 2 & 3 & 4 & 5 & 6 & 7 & 8 & 9 & 10 & 11 & 12 & 13 & 14 & 15 \\
\hline P1 & 1,01 & 0,51 & 0,34 & 0,25 & 0,2 & 0,17 & 0,14 & 0,13 & 0,11 & 0,1 & 0,09 & 0,08 & 0,08 & 0,07 & 0,07 \\
P2 & 1,01 & 0,51 & 0,34 & 0,25 & 0,2 & 0,17 & 0,14 & 0,13 & 0,11 & 0,1 & 0,09 & 0,08 & 0,08 & 0,07 & 0,07 \\
P3 & 1,01 & 0,51 & 0,34 & 0,25 & 0,2 & 0,17 & 0,14 & 0,13 & 0,11 & 0,1 & 0,09 & 0,08 & 0,08 & 0,07 & 0,07 \\
P3L3 & $-5,2$ & $-2,6$ & $-1,7$ & $-1,3$ & -1 & $-0,9$ & $-0,7$ & $-0,7$ & $-0,6$ & $-0,5$ & $-0,5$ & $-0,4$ & $-0,4$ & $-0,4$ & $-0,3$ \\
\hline
\end{tabular}


Tabela 6. Parâmetros obtidos da regressão polinomial de $3^{\circ}$ grau aplicada aos valores de espessura do pericôndio.

\begin{tabular}{|c|c|c|c|c|c|c|c|c|c|}
\hline & P1L1 & P1L2 & P1L3 & P2L1 & P2I2 & P213 & P3L1 & P3L2 & P3L3 \\
\hline$a$ & $\begin{array}{c}1,28 \mathrm{E}-0,3 \\
\pm \\
9,66 \mathrm{E}-04\end{array}$ & $\begin{array}{c}3,23 \mathrm{E}-03 \\
\pm \\
1,10 \mathrm{E}-03\end{array}$ & $\begin{array}{c}3,94 / \mathrm{E}-03 \\
\pm \\
8,81 \mathrm{E}-04 \\
\end{array}$ & $\begin{array}{c}1,24 \mathrm{E}-03 \\
\pm \\
1,47 \mathrm{E}-03\end{array}$ & $\begin{array}{c}1,83 \mathrm{E}-03 \\
\pm \\
1,23 \mathrm{E}-03 \\
\end{array}$ & $\begin{array}{c}3,03 \mathrm{E}-03 \\
\pm \\
7,79 \mathrm{E}-04\end{array}$ & $\begin{array}{c}1,43 \mathrm{E}-04 \\
\pm \\
6,92 \mathrm{E}-04\end{array}$ & $\begin{array}{c}2,25 \mathrm{E}-03 \\
\pm \\
1,46 \mathrm{E}-03 \\
\end{array}$ & $\begin{array}{c}1,43 \mathrm{E}-03 \\
\pm \\
5,60 \mathrm{E}-04\end{array}$ \\
\hline$C$ & $6,89 \pm 1,97$ & $7,97 \pm 2,25$ & $11,79 \pm 1,80$ & $4,76 \pm 3,00$ & $5,70 \pm 2,51$ & $7,73 \pm 1,59$ & $2,76 \pm 1,41$ & $7,87 \pm 3,19$ & $2,70 \pm 1,14$ \\
\hline$d$ & $68,3 \pm 1,97$ & $68,5 \pm 8,7$ & $59,2 \pm 6,9$ & $78,9 \pm 11,5$ & $78,0 \pm 9,7$ & $2,76 \pm 1,41$ & $68,1 \pm 5,4$ & $72,3 \pm 12,3$ & $70,5 \pm 4,4$ \\
\hline Nota: & $\begin{array}{l}\text { a Coeficien } \\
\text { b Coeficien } \\
\text { d Coeficien }\end{array}$ & $\begin{array}{l}\text { de grau } 3 \\
\text { de grau } 2 \\
\text { de grau } 1\end{array}$ & & & ** & $\begin{array}{l}\text { gnificante ao } \\
\text { gnificante ao }\end{array}$ & $\begin{array}{l}\text { vel de } 1 \% \\
\text { vel de } 5 \%\end{array}$ & & \\
\hline
\end{tabular}

a cartilagem septal e a junção da cartilagem septal com a placa perpendicular do etmóide. Nossos achados quanto às observações histológicas mostraram, em indivíduo com seis anos de idade, que as características histológicas de crescimento são bem menos nítidas quando comparadas com indivíduos mais jovens como quatro meses e nove meses: há poucas células na periferia, zona de transição menos exuberante e formação de grupos isógenos dispersos no centro da cartilagem, semelhantes a indivíduos com 12, 15 e 37 anos. A atividade metabólica na matriz sofre queda contínua e regular com a idade, com exceção de um caso (P2L2), ocorreu queda linear desde o nascimento até idade avançada. Aparentemente, a região P2L2, por conter o volume mais central de cartilagem, deve atingir mais rapidamente os níveis de maturação que as demais regiões. Isto explicaria o fato de não ter diferença significativa entre as várias idades. Trabalhos de Vetter et al ${ }^{27,29}$ utilizando técnicas "in vitro" mostraram diferenças de atividades metabólicas em diferentes regiões do septo nasal, mostrando declínio idade dependente e apontaram regiões que deveriam ser preservadas ou ter mínima ressecção como a borda livre anterior do septo, região supra pré-maxilar e a região central. Para a avaliação da atividade metabólica da matriz utilizamos métodos diferentes destes autores, salientando que nosso estudo ocorreu "in vivo". Nossos achados não mostraram atividade metabólica da cartilagem significativamente diferentes em todas as áreas estudadas, nas diferentes idades, porém também obtivemos queda de uma forma linear da atividade metabólica com o envelhecer do indivíduo, sendo alta na infância, menor na adolescência e bem menor na fase adulta. Baseado em nossos achados, as ressecções cirúrgicas podem ser realizadas de maneira semelhante em qualquer área da cartilagem, uma vez que não detectamos diferenças de atividades metabólicas entre as posições estudadas. No que diz respeito à espessura da cartilagem, pelo menos em cinco regiões da cartilagem septal foi possível obter uma curva dos valores esperados da espessura da cartilagem por idade. Esta curva foi capaz de descrever o comportamento de todas as medidas da espessura por posição. Em todas as curvas dos valores da espessura do septo nas três posições foi ajustada com nível de significância $\leq 5 \%$. A velocidade de crescimento em todas as idades contidas no intervalo de 0 a 15 anos foi obtida derivando-se a função que descreve o crescimento para cada idade. Sabendo que a maioria dos desvios está situado na região caudal e que uma velocidade de crescimento de espessura menor que 1\% é atingida a partir dos nove anos, a cirurgia do septo poderia ser realizada com maior segurança dos 9 aos 15 anos para as regiões: caudal, central (superior) e central (inferior), uma vez que é baixa a velocidade de crescimento da espessura encontrada nessas áreas. Nas situações nas quais a velocidade é maior que $1 \%$, com idades variando de 5 a 8 anos podemos realizar a cirurgia com cautela, visto que neste período a cartilagem septal ainda está com crescimento considerável. Isto ocorre nas regiões caudal e central anterior (superior). O crescimento da cartilagem septal teria papel ativo no crescimento facial empurrando a maxila para frente e para baixo ${ }^{30}$. Outros autores acreditavam que o septo desempenhava um papel passivo no crescimento facial. Esta discordância gerou dúvidas sobre a intervenção cirúrgica do septo em indivíduos em fase de crescimento. Nossos resultados mostraram que a velocidade de crescimento do comprimento do septo é maior na idade de um a três anos nas três posições estudadas e vai diminuindo com o passar dos anos, embora ainda seja significativo até os cinco anos. Dessa maneira parece-nos que intervenções cirúrgicas devem ocorrer após os cinco anos de idade quando a velocidade de crescimento do comprimento do septo já é menor. Entretanto, é a partir dos oito anos que a velocidade de crescimento atinge seus menores valores, fato este que indica ocasião melhor para se iniciar as intervenções cirúrgicas septais. Nossas considerações são concordantes com as de Olsen et a ${ }^{18}$ que preconizaram que o septo nasal intacto é necessário para o desenvolvimento facial normal. Estes autores preferiram procedimentos de reconstrução ao invés de ressecção radical, apontaram que nenhuma modificação do crescimento nasal ocorreu no pós-operatório. Também Jennes \& Conn $^{17}$ fizeram 
colocações semelhantes afirmando que cirurgias conservadoras, com mínimas ressecções mostraram desenvolvimento normal do nariz no acompanhamento pós-operatório. O crescimento do pericôndrio não se comportou da mesma maneira que o crescimento em espessura e em comprimento da cartilagem septal. A espessura máxima alcançada variou conforme a posição, local e idade. Portanto, o comportamento do pericôndrio no sentido de aumento de sua espessura não deve ser o parâmetro principal sobre a idade da intervenção cirúrgica. Verificamos que a idade mínima de maior espessura do pericôndrio foi observada aos 13 anos para a posição cefálica (posterior), aos 17 anos apara a posição média e aos 16 anos para a posição caudal. A importância de sua preservação cirúrgica se deve principalmente ao seu papel de nutrição da cartilagem septal. A cirurgia septal pode ser realizada a partir da idade de seis anos e com maior segurança de oito para nove anos. O dictum "esperem até o paciente completar 16 ou 17 anos para então submetê-lo a cirurgia septal ${ }^{14 "}$ parece ser uma medida de superproteção, muito provavelmente pelos temores advindos de situações onde ocorram complicações, devido principalmente à falta de experiência cirúrgica, ou à utilização de métodos cirúrgicos radicais.

\section{CONCLUSÃO}

O estudo morfométrico da espessura e do comprimento da cartilagem septal no período de 2 dias a 66 anos de idade mostrou maior velocidade de crescimento nos primeiros cinco anos de idade. Essa velocidade vai diminuindo com a idade. O estudo da atividade metabólica da cartilagem através da densitometria mostrou que o tecido apresenta comportamento semelhante em toda sua extensão, sendo mais alta nos primeiros anos de vida. Com essas observações consideramos que as intervenções cirúrgicas podem ser realizadas após esse período, quando necessárias. Entretanto, o período mais indicado para essas cirurgias inicia-se após os oito anos de idade.

\section{REFERÊNCIAS BIBLIOGRÁFICAS}

1. Blaugrund SM. The Nasal Septum and Concha Bullosa. Otolaryngo Clin North Am, 1989;22(2):291-306.

2. Metzenbaum M. Assimetry of the Nares: A Positive Diagnostic Sign or Entity Establishing Anatomic Displacement of Lower End of Cartilagenous Nasal Septum, Arch Otolaryngol, 1932;16:690-7.

3. Metzenbaum M. Dislocation of the Lower End of the Nasal Septal Cartilage. Arch Otolaryngol, 1936;24:78-85.

4. Cottle MH. Nasal Surgery in Children. EENT Monthly, $1951 ; 30: 32-8$
5. Cottle $\mathrm{MH}$ \& Loring RM. The Maxila-premaxila Approach to Extensive Nasal Septum Surgery. Arch Otolaryngol, 1958;68:301-13.

6. Gilbert JG \& Segal SL. Growth of the Nose and the septorhinoplastic Problem in youth. Arch Otolaryngol, 1958;68:673-82.

7. Gray LP. The deviated nasal septum I aetiology. J Laryngol, $1965 ; 79: 565-75$

8. Montserrat JM. Cirurgia Funcional de la Nariz em la Infância. Acta Orl Iber Am, 1968;2:150-62.

9. Stucker FJJR, Bryarly RC, Shockley WW. Management of Nasal Trauma in Children. Arch Otolaryngol, 1984;110:190-2.

10.Jugo S. Septal Surgery in Children. Symp Otorhinol Iug. 1986;21(23):29142

11. Stool SE. Postnatal Craniofacial Growth and Developement in Bluestone CD \& Stool SE. Pediatric.Otolaryngology, Philadelfia,WB SAUNDERS, 1983;1(2):18-30.

12. Montserrat-Viladiu JM \& Montserrat-Gali JR. Septoplastia Infantil. F Med (BR), 1987;95(2):83-92.

13. White FW. Submucous Resection of the Nasal Septum in Children, Arch Otolaryngol, 1930;11:415-25.

14. Salinger S. Injuries to the Nose in Children. Arch Otolaryng, 1941;34:93651.

15. Lefkon IM. Rhinoiplasty in Children. Arch Otolaryngol, 1948;48:7382.

16. Fine IJ. Goldman techinique in nasal septal surgery. Arch Otolaryngol, 1954;54:141-6.

17. Jennes ML \& Conn W. Corrective Nasal Surgery in Children; Arch Otolaryngol, 1964;79:145-51.

18. Olsen HD, Carpenter RJ, Kern EB. Nasal Septal Injury in Children, Arch Otolaryngol, 1980;106:317-20.

19. Fior R \& Veljak C. Septum dislocation in the newborn a log term follow up study of immediate reposition. Rhinology, 1990;28:159-62.

20. Walker PJ, Crysdale WS, Farkas LG. External Septorhinoplasty in Children: Outcome and Effects on Growth of Septal Excision and Reimplantation, Arch Otolaryngol Head Neck Surg, 1993;119(9):984-9.

21. Goodman WS. External Approach to Rhinoplasty. Can J Otolaryngol, 1973;2:207-10.

22. Goodman WS \& Charbnneau PA. External Approach to Rhinoplasty. Laryngoscope, 1974;84:2195-201.

23. Goodman WS \& Charle DA. Techinique of External Rhinoplasty J Otolaryngol, 1978;7(1):13-7.

24. Goodman WS. Recent Advances in External Rhinoplasty. J Otolaryngol, 1982;10:433-9.

25. Anderson JR, Johnson CM, Adamson P. Open Rhinoplasty: An Assesment, Otolaryngol Head Neck Surg, 1982;90:272-4.

26. Hall BK. Cartilage. Structure, Function and Biochemistry. EUA, Academic press, subs. Harcourt Brace Jovamovich Publ., The Chondroblast and the Chondocyte, 1983;1(3):59-78.

27. Vetter V, Heit W, Helbing G, Heinze E, Pirsig W. Growth of the Septal Cartilage: Cell Density and Colony Formation of Septal Chondrocytes. Laryngoscopy, 1984;94:1226-9.

28. Klaff DD. The Surgical Anatomy of the Antero-Caudal Portion of the Nasal Septum: A Study of the Area of the Premaxilla. Laringoscope, 1956;66(7):995-1020.

29. Vetter V, Pirsig W, Heinze E. Growth Activity in Human Septal Cartilage: Age Dependent Incorporation of Labeled Sulfate in Different Anatomic Location, Plast Reconstr Surg, 1983;71(2):167-71.

30. Scott JH. The Cartilage of the Nasal Septum, Brit Dent J, $1953 ; 95: 3743$ 